\title{
An Algorithm for SDV Representation of 2D Behaviors
}

\author{
Isabel Brás and Paula Rocha
}

\begin{abstract}
This paper deals with the characterization of 2D behaviors that are representable by means of special first order models, known as state/driving-variable (SDV) models. In previous work, [1], [2] we have shown how to identify SDV-representable behaviors using one of its full row rank representations. Here, we give a further refinement by showing that a 2D behavior is SDV-representable if and only if each of its kernel representations can be decomposed as a product of three 2D L-polynomial matrices: a zero right prime matrix, a cw-unital square matrix and a factor left prime matrix. Using that decomposition, we present a procedure to obtain SDV representations of a 2D behavior starting from any of its kernel representations.
\end{abstract}

\section{INTRODUCTION}

In this paper we consider the class of $2 \mathrm{D}$ behaviors $\mathcal{B}$ that can be described as the solution set of a system of linear partial difference equations of the form

$$
R\left(\sigma_{1}, \sigma_{2}, \sigma_{1}^{-1}, \sigma_{2}^{-1}\right) w=0
$$

where

$$
R\left(\sigma_{1}, \sigma_{2}, \sigma_{1}^{-1}, \sigma_{2}^{-1}\right)=\sum_{(i, j) \in S} R_{i j} \sigma_{1}^{i} \sigma_{2}^{j},
$$

with $S \subset \mathbb{Z}^{2}$ finite, is a 2D Laurent-polynomial (Lpolynomial) shift operator, $\sigma_{1}$ and $\sigma_{2}$ are the usual 2D shifts, (i.e. $\sigma_{1} x(i, j)=x(i+1, j), \sigma_{2} x(i, j)=x(i, j+$ $1), \forall(i, j) \in \mathbb{Z}^{2}$ and $\left.\forall x: \mathbb{Z}^{2} \rightarrow \mathbb{R}^{n}\right)$, and the system variable $w$ is a vector valued signal whose components are not divided into inputs and outputs. For short, since $\mathcal{B}=$ ker $R\left(\sigma_{1}, \sigma_{2}, \sigma_{1}^{-1}, \sigma_{2}^{-1}\right)$, we shall refer to such behaviors as kernel behaviors.

Note that $R\left(\sigma_{1}, \sigma_{2}, \sigma_{1}^{-1}, \sigma_{2}^{-1}\right)$ may be a higher order operator, since no limits are imposed on the degrees of monomials $\sigma_{1}^{k_{1}} \sigma_{2}^{k_{2}}$. The question that we investigate is the existence and construction of an alternative description which is first order, in the sense that at each point in the grid $(i, j)$ is updated using the state and the driving-variable values at the two nearest neighbors $(i-1, j)$ and $(i, j-1)$. More concretely we are interested in representations of the form

$$
\left\{\begin{aligned}
x & =A\left(\sigma_{1}^{-1}, \sigma_{2}^{-1}\right) x+B\left(\sigma_{1}^{-1}, \sigma_{2}^{-1}\right) v \\
w & =C x+D v
\end{aligned}\right.
$$

This work was partially supported by the Unidade de Investigação Matemática e Aplicações (UIMA), University of Aveiro, Portugal, through the Programa Operacional "Ciência e Tecnologia e Inovação"(POCTI) of the Fundação para a Ciência e Tecnologia (FCT), co-financed by the European Union fund FEDER

Isabel Brás is with the Department of Mathematics, University of Aveiro, Campo Universitário de Santiago, 3810-193 Aveiro, Portugal, isabelb@mat . ua.pt

Paula Rocha is with the Department of Mathematics, University of Aveiro, Campo Universitário de Santiago, 3810-193 Aveiro, Portugal, procha@mat.ua.pt where $x$ and $v$ are auxiliary variables, $A\left(s_{1}^{-1}, s_{2}^{-1}\right)=$ $A_{1} s_{1}^{-1}+A_{2} s_{2}^{-1}, B\left(s_{1}^{-1}, s_{2}^{-1}\right)=B_{1} s_{1}^{-1}+B_{2} s_{2}^{-1}$ and $A_{1}, A_{2}, B_{1}, B_{2}, C$ and $D$ are real matrices of suitable dimensions. From the formal point of view, SDV models are very similar to the well-known Fornasini-Marchesini models, [3], but with the essential difference that here the role of the input is taken by the driving-variable, while the system variable $w$ (which contains both inputs and outputs) plays the role of an output.

In previous work, [1], we have shown that SDVrepresentability is equivalent to the existence of a kernel representation that can be factored as a product of a leftprime 2D L-polynomial matrix by a square 2D L-polynomial matrix with 2D-proper inverse. This factorization condition emphasizes the existence a certain SDV-representable autonomous part of the behavior. Indeed, the kernel of the above mentioned square matrix is an SDV-representable autonomous part. Since the same behavior may have distinct autonomous parts with different representability properties, see [2], it seems suitable to present a SDV-representability characterization which does not rest on the properties of a certain autonomous part. Results in this direction have been obtained in [2]. Indeed, we have shown that a 2D behavior $\mathcal{B}$ is SDV-representable if and only if it has a full row rank kernel representation and, additionally, the gcd's of the maximal order minors of any full row rank kernel representation of $\mathcal{B}$ are unimodularly equivalent to a $2 \mathrm{D}$ L-polynomial with 2D-proper inverse. Here we reformulate this characterization in terms of the concept of cw-unital polynomials and obtain a further refinement. Furthermore, this is done using a different line of reasoning.

It is a well-known fact that every 2D L-polynomial matrix can be factored as a product of three matrices: a factor right prime matrix by a square non-singular matrix by a factor left prime matrix. We will show that SDV-representable behaviors are exactly the ones for which this decomposition of its kernel representations yields factorizations where the factor right matrix is indeed a zero right prime and the square matrix has cw-unital determinant. This result provides a way of deciding whether or not a kernel behavior is SDVrepresentable using any of its kernel representations.

Our approach to this representability problem allows to present a procedure for the construction of SDVrepresentations for a $2 \mathrm{D}$ behavior that can be performed starting from any of its kernel representations. 


\section{PReliminaries}

In the following we consider discrete $2 \mathrm{D}$ systems $\Sigma=\left(\mathbb{Z}^{2}, \mathbb{R}^{q}, \mathcal{B}\right)$ that admit a kernel representation, i.e.,

$$
\mathcal{B}=\operatorname{ker} R\left(\sigma_{1}, \sigma_{2}, \sigma_{1}^{-1}, \sigma_{2}^{-1}\right) .
$$

The matrix $R\left(s_{1}, s_{2}, s_{1}^{-1}, s_{2}^{-1}\right)$ is simply said to be a representation of $\Sigma$ (and $\mathcal{B})$. We denote the set of all 2D systems $\Sigma=\left(\mathbb{Z}^{2}, \mathbb{R}^{q}, \mathcal{B}\right)$ with kernel representation by $\mathcal{L}^{q}$. Note that, a $2 \mathrm{D}$ system $\Sigma \in \mathcal{L}^{q}$ has infinitely many representations. In fact, $R$ and $\tilde{R}$ are representations of $\Sigma$ if and only if there exist L-polynomial matrices $L$ and $\tilde{L}$, of suitable dimensions, such that $R=L \tilde{R}$ and $\tilde{R}=\tilde{L} R$, [4]. When two Lpolynomial matrices represent the same behavior they are said to be equivalent. If $L$ is unimodular, which means that $L$ is invertible as a L-polynomial matrix, $R$ and $\tilde{R}$ are said to be unimodularly equivalent (note that in this case $\tilde{L}$ is the inverse of $L$ ). For instance, two full row rank representations of the same behavior are unimodularly equivalent. Notice that not every behavior has a full row rank representation. $\mathcal{B}$ is said to be a regular behavior if it admits a full row rank representation.

Definition 1: Let $\Sigma=\left(\mathbb{Z}^{2}, \mathbb{R}^{q}, \mathcal{B}\right) \in \mathcal{L}^{q}$. The system of equations

$$
\left\{\begin{aligned}
x & =A\left(\sigma_{1}^{-1}, \sigma_{2}^{-1}\right) x+B\left(\sigma_{1}^{-1}, \sigma_{2}^{-1}\right) v \\
w & =C x+D v
\end{aligned}\right.
$$

where $\sigma_{1}, \sigma_{2}$ are the usual 2D shifts,

$$
\begin{aligned}
& A\left(s_{1}^{-1}, s_{2}^{-1}\right)=A_{1} s_{1}^{-1}+A_{2} s_{2}^{-1} \in \mathbb{R}^{n \times n}\left[s_{1}^{-1}, s_{2}^{-1}\right], \\
& B\left(s_{1}^{-1}, s_{2}^{-1}\right)=B_{1} s_{1}^{-1}+B_{2} s_{2}^{-1} \in \mathbb{R}^{n \times m}\left[s_{1}^{-1}, s_{2}^{-1}\right], \\
& C \in \mathbb{R}^{q \times n} \text { and } D \in \mathbb{R}^{q \times m},
\end{aligned}
$$

is called a state/driving-variable representation (SDV) of $\Sigma$ (of $\mathcal{B}$ ) if

$$
\mathcal{B}=\left\{w: \mathbb{Z}^{2} \rightarrow \mathbb{R}^{q} \mid \exists x, v \text { such that (2) holds }\right\} .
$$

In this case $\mathcal{B}$ is said to be SDV-representable.

As mentioned in the Introduction, the question of the existence of such a representation has already been studied in [1], [2]. Let us recall some basic facts, already shown in that previous work, which are here our starting point.

It is a well-known fact that every $2 \mathrm{D}$ behavior is decomposable as a sum of its controllable part, $\mathcal{B}^{c}$, with an autonomous part, [5]. Taking this into account together with the fact that controllable behaviors are always SDVrepresentable, [4], it is simple to conclude the following, as shown in [1].

Proposition 1: Let $\mathcal{B}$ be a $2 \mathrm{D}$ kernel behavior. If $\mathcal{B}$ has an SDV-representable autonomous part, then $\mathcal{B}$ is SDVrepresentable.

Notice that, the reciprocal implication of the Proposition 1 holds, as shown in [1]. However, in this approach we need not use this implication and, naturally, it comes as a consequence of the results presented in the sequel (see Remark 2).
The SDV-representability of an autonomous behavior is characterized in [1], [2] in terms of the notion of 2Dproperness. We say that a $2 \mathrm{D}$ rational function $f$ is $2 \mathrm{D}$ proper if $f=p / q$, where $p, q \in \mathbb{R}\left[s_{1}^{-1}, s_{2}^{-1}\right]$ and the zerodegree coefficient of $q$ is nonzero. A 2D rational matrix will be called 2D-proper if all its entries are 2D-proper rational functions. Recall that every $2 \mathrm{D}$ L-polynomial matrix $R\left(s_{1}, s_{2}, s_{1}^{-1}, s_{2}^{-1}\right)$ can be written as

$$
R\left(s_{1}, s_{2}, s_{1}^{-1}, s_{2}^{-1}\right)=\sum_{(i, j) \in S} R_{i j} s_{1}^{i} s_{2}^{j},
$$

where $S$ is a finite subset of $\mathbb{Z}^{2}$ and $R_{i j}$ is a nonzero constant matrix, for $(i, j) \in S$. The set $S$ is the support of $R$, usually denoted by $\operatorname{supp}(R)$. Notice that, with this notation the definition of $2 \mathrm{D}$-properness given above may be reformulated by saying that $p$ and $q$ have their supports in the third quarter plane and moreover the zero-degree coefficient of $q$ is nonzero.

In fact, in [1] we have shown the following result.

Proposition 2: Let $\Sigma=\left(\mathbb{Z}^{2}, \mathbb{R}^{q}, \mathcal{B}\right) \in \mathcal{L}^{q}$ be an autonomous system. $\mathcal{B}$ is SDV-representable if and only if there exists a kernel representation of $\mathcal{B}$ with $2 \mathrm{D}$-proper inverse.

Since every two square representations of an autonomous behavior are unimodularly equivalent we may state the next corollary.

Corollary 1: Let $\Sigma=\left(\mathbb{Z}^{2}, \mathbb{R}^{q}, \mathcal{B}\right) \in \mathcal{L}^{q}$ be an autonomous system. $\mathcal{B}$ is SDV-representable if and only if $\mathcal{B}$ is regular and every square representation of $\mathcal{B}$ is unimodularly equivalent to a square L-polynomial matrix with $2 \mathrm{D}$-proper inverse.

It is possible to identify a 2D square L-polynomial matrix unimodularly equivalent to a square L-polynomial matrix with 2D-proper inverse by its determinant. Indeed, the following has been proven in [2].

Lemma 1: A 2D L-polynomial square matrix $M$ is unimodularly equivalent to a square L-polynomial matrix with 2D-proper inverse if and only if $\operatorname{det} M$ is unimodularly equivalent to a 2D L-polynomial with $2 \mathrm{D}$-proper inverse.

As an immediate consequence of Corollary 1 and Lemma 1 it is possible to state the next corollary.

Corollary 2: An autonomous system $\mathcal{B}$ is SDVrepresentable if an only if $\mathcal{B}$ is regular and every square representation is such that its determinant is unimodularly equivalent to a $2 \mathrm{D}$ L-polynomial with $2 \mathrm{D}$-proper inverse. 


\section{REPRESENTABLE AUTONOMOUS BEHAVIORS}

Let us consider the componentwise order (cw-order) in $\mathbb{Z}^{2}$, [6], given by

$$
\begin{gathered}
\left(m_{1}, m_{2}\right) \leq \mathrm{cw}\left(n_{1}, n_{2}\right) \\
\quad \text { if and only if } \\
m_{1} \leq n_{1} \wedge m_{2} \leq n_{2} .
\end{gathered}
$$

Notice that $\leq_{\mathrm{cw}}$ is a partial order of $\mathbb{Z}^{2}$. So, as expected, given a L-polynomial matrix $R$ it may not exist $\left(d_{1}, d_{2}\right) \in$ $\operatorname{supp}(R)$ such that $\left(d_{1}, d_{2}\right) \leq \mathrm{cw}(i, j), \forall(i, j) \in \operatorname{supp}(R)$. This observation gives rise to the following definitions:

\section{Definition 2:}

(i) A L-polynomial $p \in \mathbb{R}\left[s_{1}, s_{2}, s_{1}^{-1}, s_{2}^{-1}\right]$ is cw-unital if $p$ has the form

$$
p=p_{d_{1} d_{2}} s_{1}^{d_{1}} s_{2}^{d_{2}}+\sum_{(i, j)<\mathrm{cw}\left(d_{1}, d_{2}\right)} p_{i j} s_{1}^{i} s_{2}^{j},
$$

where $p_{d_{1} d_{2}}$ is nonzero, [6].

(ii) A square L-polynomial matrix $R \in$ $\mathbb{R}^{g \times g}\left[s_{1}, s_{2}, s_{1}^{-1}, s_{2}^{-1}\right]$ is cw-unital if $R$ has the form

$$
R=R_{d_{1} d_{2}} s_{1}^{d_{1}} s_{2}^{d_{2}}+\sum_{(i, j)<\mathrm{cw}\left(d_{1}, d_{2}\right)} R_{i j} s_{1}^{i} s_{2}^{j},
$$

where $R_{d_{1} d_{2}}$ is invertible.

Remark 1: The previous definitions may be rewritten in the following manner. Let $S$ be a finite subset of $\mathbb{Z}^{2}$ and denote by $\sup _{c w}(S)$ the supremum of $S$ with respect to the componentwise order defined by (3). It is clear that,

(i) A L-polynomial $p$ is cw-unital if and only if $\sup _{c w}(\operatorname{supp}(p)) \in \operatorname{supp}(p)$;

(ii) A L-polynomial square matrix $R$ is cw-unital if and only if $\sup _{c w}(\operatorname{supp}(R))=\left(d_{1}, d_{2}\right) \in \operatorname{supp}(R)$ and the correspondent coefficient $R_{d_{1} d_{2}}$ is invertible.

In fact, every cw-unital L-polynomial is unimodularly equivalent to a L-polynomial with proper inverse and viceversa.

Lemma 2: A L-polynomial $p$ is cw-unital if and only if $p$ is unimodularly equivalent to a polynomial with proper inverse.

Proof: $p$ is cw-unital if and only if

$$
p=p_{d_{1} d_{2}} s_{1}^{d_{1}} s_{2}^{d_{2}}+\sum_{(i, j)<\mathrm{cw}\left(d_{1}, d_{2}\right)} p_{i j} s_{1}^{i} s_{2}^{j},
$$

where $p_{d_{1} d_{2}}$ is nonzero, i.e., if and only if

$$
s_{1}^{-d_{1}} s_{2}^{-d_{2}} p=p_{d_{1} d_{2}}+\sum_{(i, j)<\mathrm{cw}(0,0)} p_{i j} s_{1}^{i} s_{2}^{j},
$$

where $p_{d_{1} d_{2}}$ is nonzero, which means that $p$ is unimodularly equivalent to a polynomial with proper inverse.

Notice that not every matrix that is unimodularly equivalent to a matrix with proper inverse is $\mathrm{cw}$-unital. However, it is easy to show the following lemma (for the less obvious equivalence, (ii) $\Leftrightarrow$ (iii), see [1]).

Lemma 3: Let $\mathrm{R}$ be a square 2D L-polynomial matrix. The following statements are equivalent:

(i) $\operatorname{det} R$ is cw-unital;

(ii) $R$ is unimodularly equivalent to a $2 \mathrm{D}$ L-polynomial matrix with $2 \mathrm{D}$ proper inverse;

(iii) $R$ is unimodularly equivalent to a $2 \mathrm{D}$ L-polynomial matrix with support in the third quarter plane and invertible independent term.

(iv) $R$ is unimodularly equivalent to a cw- unital 2D Lpolynomial matrix

Considering Lemma 2, Corollary 2 may be restated in the following nicer way.

Proposition 3: An autonomous system $\mathcal{B}$ is SDVrepresentable if an only if $\mathcal{B}$ is regular and every square representation is such that its determinant is cw-unital.

\section{General RePresentability}

In this section we consider systems $\Sigma=\left(\mathbb{Z}^{2}, \mathbb{R}^{q}, \mathcal{B}\right) \in$ $\mathcal{L}^{q}$, that are not necessarily autonomous, and show how to obtain the SDV-representability characterization correspondent to the one given in Proposition 3. This is made based on a factorization that emphasizes the controllable part of the behavior.

The following lemma is basically a reformulation of Lemma A.1 in [7] and this was pointed out to us by E. Zerz.

Lemma 4: Let $R_{1} \in \mathbb{R}^{g_{1} \times q}\left[s_{1}, s_{2}, s_{1}^{-1}, s_{2}^{-1}\right]$ and $R_{2} \in$ $\mathbb{R}^{g_{2} \times q}\left[s_{1}, s_{2}, s_{1}^{-1}, s_{2}^{-1}\right]$. Then $\operatorname{ker} R_{1}+\operatorname{ker} R_{2}=\operatorname{ker} L$, where $L$ is a least common left factor of $R_{1}$ and $R_{2}$. that

Proof: Let $L \in \mathbb{R}^{g \times q}\left[s_{1}, s_{2}, s_{1}^{-1}, s_{2}^{-1}\right]$ be a matrix such

$$
\operatorname{ker} R_{1}+\operatorname{ker} R_{2}=\operatorname{ker} L .
$$

Note that such a L-polynomial matrix exists, see for instance [8]. Thus, we shall show that $L$ is a least common left factor of $R_{1}$ and $R_{2}$. In fact, $w \in \operatorname{ker} L$ iff there exist $w_{1} \in \operatorname{ker} R_{1}$ and $w_{2} \in \operatorname{ker} R_{2}$ such that $w=w_{1}+w_{2}$. That is, $w \in \operatorname{ker} L$ iff there exist $w_{1} \in \operatorname{ker} R_{1}$ and $w_{2} \in \operatorname{ker} R_{2}$ such that

$$
\left[\begin{array}{cc}
I & I \\
R_{1} & 0 \\
0 & R_{2}
\end{array}\right]\left[\begin{array}{l}
w_{1} \\
w_{2}
\end{array}\right]=\left[\begin{array}{l}
I \\
0
\end{array}\right] w .
$$

So, (4) is a representation of $\operatorname{ker} L$ with auxiliary variables $w_{1}$ and $w_{2}$. Then, there exist L-polynomial matrices $F_{1}$ and $F_{2}$ such that the L-polynomial matrix

$$
\left[\begin{array}{lll}
L & -F_{1} & -F_{2}
\end{array}\right]
$$

is a minimal left annihilator of

$$
\left[\begin{array}{cc}
I & I \\
R_{1} & 0 \\
0 & R_{2}
\end{array}\right]
$$


That is equivalent to say that $L=F_{1} R_{1}, L=F_{2} R_{2}$ and if

$$
\left[\begin{array}{lll}
\bar{L} & -\bar{F}_{1} & -\bar{F}_{2}
\end{array}\right]\left[\begin{array}{cc}
I & I \\
R_{1} & 0 \\
0 & R_{2}
\end{array}\right]=0
$$

then

$$
\left[\begin{array}{lll}
\bar{L} & -\bar{F}_{1} & -\bar{F}_{2}
\end{array}\right]=T\left[\begin{array}{lll}
L & -F_{1} & -F_{2}
\end{array}\right]
$$

for some L-polynomial matrix $T$. Therefore, $L$ is a least common left factor of $R_{1}$ and $R_{2}$.

It is a well-known fact that a full row rank L-polynomial matrix may be factored as a product of a square matrix by factor left prime one. In the following lemma we establish a relation between the determinant of such a square matrix and a representation of a certain autonomous part of the behavior.

Lemma 5: If $\mathcal{B}=\operatorname{ker}\left(\Delta H_{c}\right)$, where $\Delta$ is a nonsingular square 2D L-polynomial and $H_{c}$ is a factor left prime one, then there exists an autonomous part of $\mathcal{B}, \widehat{\mathcal{B}}^{a}$, such that $\widehat{\mathcal{B}}^{a}=\operatorname{ker} R^{a}$, where $\operatorname{det} R^{a}=\operatorname{det}(\Delta) \cdot d\left(s_{1}\right)$, with $d\left(s_{1}\right)$ a polynomial in $s_{1}$.

Proof: First of all notice that $H_{c}$ is a representation of the controllable part of $\mathcal{B}$, see for instance [5]. Let $H=$ $\Delta H_{c}$. Since $H_{c}$ is factor left prime, there exist $C_{1}$ and $C_{2}$ such that

$$
\operatorname{det}\left[\begin{array}{c}
H_{c} \\
C_{i}
\end{array}\right]=d_{i}\left(s_{i}\right), i=1,2,
$$

where $d_{1}\left(s_{1}\right)$ and $d_{2}\left(s_{2}\right)$ are nonzero polynomials in $s_{1}$ and $s_{2}$, respectively, [9].

Notice that

$$
\operatorname{ker} H \cap \operatorname{ker} C_{1}=\operatorname{ker}\left(\left[\begin{array}{cc}
\Delta & 0 \\
0 & I
\end{array}\right]\left[\begin{array}{l}
H_{c} \\
C_{1}
\end{array}\right]\right) \text {. }
$$

Let $R^{a}:=\left[\begin{array}{cc}\Delta & 0 \\ 0 & I\end{array}\right]\left[\begin{array}{l}H_{c} \\ C_{1}\end{array}\right]$. We next show that

$$
\widehat{\mathcal{B}}^{a}=\operatorname{ker} R^{a}
$$

is an autonomous part of $\mathcal{B}$. By Lemma 4

$$
\text { ker } H_{c}+\operatorname{ker} R^{a}=\operatorname{ker} L,
$$

where $L$ is a l.c.l.f. $\left(H_{c}, R^{a}\right)$. In order to determine a l.c.l.f. $\left(H_{c}, R^{a}\right)$, we identify the multiples of $H_{c}$ and $R^{a}$ as being the matrices of the form

$$
L_{0} H_{c} \text {, for some matrix } L_{0},
$$

and

$$
L_{1} \Delta H_{c}+L_{2} C_{1} \text {, for some matrices } L_{1}, L_{2},
$$

respectively. Therefore the common left multiples of $H_{c}$ and $R^{a}$ are matrices of the previous forms for which $L_{0}, L_{1}, L_{2}$ are such that

$$
L_{0} H_{c}=L_{1} \Delta H_{c}+L_{2} C_{1}
$$

that is,

$$
\left[\begin{array}{cc}
L_{0}-L_{1} \Delta & -L_{2}
\end{array}\right]\left[\begin{array}{l}
H_{c} \\
C_{1}
\end{array}\right]=0 \text {. }
$$

By construction $\left[\begin{array}{c}H_{c} \\ C_{1}\end{array}\right]$ is full row rank. Hence, $L_{0}=L_{1} \Delta$, and $L_{2}=0$, and the common left multiples of $H_{c}$ and $R^{a}$ are of the form $L_{1} \Delta H_{c}$. Therefore, $L=\Delta H_{c}$ is a l.c.l.f. $\left(H_{c}, R^{a}\right)$. Since ker $H_{c}$ is the controllable part of $\mathcal{B}$, from equation (5) we conclude that $\operatorname{ker} R^{a}$ is an autonomous part of $\mathcal{B}$. Furthermore, by construction, $R^{a}$ is square and $\operatorname{det} R^{a}=\operatorname{det}(\Delta) \cdot d\left(s_{1}\right)$, with $d\left(s_{1}\right)$ a polynomial in $s_{1}$.

Next we give a characterization of SDV-representable 2D behaviors. Clearly, Proposition 3 is contained in the following one.

Proposition 4: Let $\Sigma=\left(\mathbb{Z}^{2}, \mathbb{R}^{q}, \mathcal{B}\right) \in \mathcal{L}^{q}$. $\mathcal{B}$ is SDVrepresentable if and only if $\mathcal{B}$ is regular and every full row rank kernel representation $R$ of $\mathcal{B}$ is such that $R=\Delta H_{c}$, where $H_{c}$ is factor left prime and $\Delta$ is square with cw-unital determinant.

Proof: Assume that $\mathcal{B}$ is SDV-representable. That is, there exists a representation of $\mathcal{B}$ of the following form

$$
\left\{\begin{array}{rl}
x & =A\left(\sigma_{1}^{-1}, \sigma_{2}^{-1}\right) x+B\left(\sigma_{1}^{-1}, \sigma_{2}^{-1}\right) v \\
w & =C x+D v
\end{array} .\right.
$$

So, $\mathcal{B}$ is described by an latent variable representation as follows, [4], [10],

$$
\left[\begin{array}{l}
0 \\
I
\end{array}\right] w=\left[\begin{array}{cc}
I-A\left(\sigma_{1}^{-1}, \sigma_{2}^{-1}\right) & -B\left(\sigma_{1}^{-1}, \sigma_{2}^{-1}\right) \\
C & D
\end{array}\right]\left[\begin{array}{l}
x \\
v
\end{array}\right] .
$$

In order to eliminate the latent variables $\operatorname{col}(x, v)$, let $\left[\begin{array}{ll}L_{1} & L_{2}\end{array}\right]$ be a minimal left annihilator of

$$
\left[\begin{array}{cc}
I-A\left(\sigma_{1}^{-1}, \sigma_{2}^{-1}\right) & -B\left(\sigma_{1}^{-1}, \sigma_{2}^{-1}\right) \\
C & D
\end{array}\right] .
$$

Thus, $\mathcal{B}=\operatorname{ker} L_{2}$.

On the other hand, $\left[\begin{array}{ll}L_{1} & L_{2}\end{array}\right]$ is a left annihilator of

$$
\left[\begin{array}{c}
I-A\left(\sigma_{1}^{-1}, \sigma_{2}^{-1}\right) \\
C
\end{array}\right] \text {. }
$$

Therefore, there exists a L-polynomial matrix $F$ such that

$$
\left[\begin{array}{ll}
L_{1} & L_{2}
\end{array}\right]=F\left[\begin{array}{ll}
M_{1} & M_{2}
\end{array}\right]
$$

where $\left[\begin{array}{cc}M_{1} & M_{2}\end{array}\right]$ is a minimal left annihilator of $\left[\begin{array}{c}I-A\left(\sigma_{1}^{-1}, \sigma_{2}^{-1}\right) \\ C\end{array}\right]$. Since $\left[\begin{array}{ll}L_{1} & L_{2}\end{array}\right]$ is factor left prime, $F$ is also factor left prime. Note also that, by construction, $M_{2}$ is a square matrix and its determinant is a divisor of $\operatorname{det}\left(I-A\left(\sigma_{1}^{-1}, \sigma_{2}^{-1}\right)\right)$. Since $\operatorname{det}\left(I-A\left(\sigma_{1}^{-1}, \sigma_{2}^{-1}\right)\right)$ is a cw-unital L-polynomial and every divisor of a cw-unital L-polynomial is also a cw-unital L-polynomial (this is a consequence of $\left[6\right.$, p. 116]), we conclude that $\operatorname{det} M_{2}$ is cw-unital. Thus

$$
L_{2}=F M_{2}
$$

where $F$ is factor left prime and $\operatorname{det} M_{2}$ is cw-unital. Moreover, it is possible to prove from this that $L_{2}$ has the desired factorization. In fact, let $R_{c}$ be a representation of 
the controllable part of $\mathcal{B}$. Since $\mathcal{B}^{c} \subset \mathcal{B}$, there exists $\Delta$ such that $\Delta R_{c}=F M_{2}$, that is,

$$
\left[\begin{array}{ll}
-F & \Delta
\end{array}\right]\left[\begin{array}{c}
M_{2} \\
R_{c}
\end{array}\right]=0
$$

But $F$ is factor left prime and consequently $\left[\begin{array}{cc}-F & \Delta\end{array}\right]$ is also left factor prime. So, $\left[\begin{array}{cc}-F & \Delta\end{array}\right]$ is a minimal left annihilator of $\left[\begin{array}{c}M_{2} \\ R_{c}\end{array}\right]$. Therefore, the determinant of the non-singular matrix $\Delta$ must divide $\operatorname{det} M_{2}$ and hence be cw-unital. Thus, we have shown the existence of a full row rank representation for $\mathcal{B}$ of the form $\Delta R_{c}$, where $\Delta$ is square with cw-unital determinant and $R_{c}$ is factor left prime. Furthermore, it is easy to see that any other full row rank representation of the same behavior also admits a similar decomposition. Indeed, if $R$ is a full row rank representation of $\mathcal{B}$, there exists an unimodular matrix $U$ such that $R=U M_{2} L_{2}$ and it is quite trivial to check that $\operatorname{det} U M_{2}$ is cw-unital.

Conversely, let us suppose that $\mathcal{B}=\operatorname{ker}\left(\Delta H_{c}\right)$, where $H_{c}$ is left factor prime and $\Delta$ is square with cw-unital determinant. According to Lemma 5, there exists an autonomous part of $\mathcal{B}, \mathcal{B}^{a}$, such that $\mathcal{B}^{a}=\operatorname{ker} R^{a}$, where $\operatorname{det} R^{a}=\operatorname{det}(\Delta) \cdot d\left(s_{1}\right)$, with $d\left(s_{1}\right)$ a polynomial in $s_{1}$. Since $\operatorname{det} \Delta$ is cw-unital it is easy to check that $\operatorname{det} R^{a}$ is also cw-unital.

Remark 2: From Proposition 4, it follows that if $\mathcal{B}$ is SDV-representable then $\mathcal{B}=\operatorname{ker} \Delta H_{c}$, where $\Delta$ is cw-unital and $H_{c}$ is factor left prime. Thus, as shown in the proof of the same proposition, it is possible to exhibit an autonomous part

$$
\mathcal{B}^{a}=\operatorname{ker}\left[\begin{array}{c}
\Delta H_{c} \\
C
\end{array}\right]
$$

such that

$$
\operatorname{det}\left[\begin{array}{c}
\Delta H_{c} \\
C
\end{array}\right]=\operatorname{det}(\Delta) d\left(s_{1}\right),
$$

where $d\left(s_{1}\right)$ is polynomial in $s_{1}$ only. Therefore, $\mathcal{B}^{a}$ is an SDV-representable autonomous part of $\mathcal{B}$. Notice that, this type of autonomous part construction was proposed in [7].

As already mentioned, every full row rank 2D Lpolynomial matrix can be factored as a product of a square non-singular matrix by a factor left prime one. It follows directly from the previous proposition that SDV-decomposable behavior are exactly those where, in every such a decomposition, the square matrix has $\mathrm{cw}$-unital determinant.

Corollary 3: If $\mathcal{B}=\operatorname{ker}(\Delta F)$, where $\Delta$ is a non-singular square matrix and $F$ is factor left prime, then $\mathcal{B}$ is SDVrepresentable if and only if $\operatorname{det} \Delta$ is cw-unital.

Notice that, according to the previous corollary, if one has a full row rank representation of behavior, in order to check its SDV-representability one may simply calculate a maximal left factor of such representation and then see whether or not its determinant is cw-unital. However, even when a behavior is regular, there are representations without full row rank. Next we shall see how such representations can be characterized for SDV-representable behaviors.

Clearly, even with the full row rank condition is dropped, every 2D L-polynomial matrix can be factored as a product of three matrices: a factor right prime matrix by a square nonsingular matrix by a left factor left prime. We will show that SDV-representable behaviors are exactly the ones for which the above mentioned decomposition yields a factorization where the factor right prime matrix is in fact zero right prime and the square matrix has cw-unital determinant.

Proposition 5: If $\mathcal{B}=\operatorname{ker}(P \Delta F)$, where $P$ is factor right prime, $\Delta$ is a non-singular square matrix and $F$ is factor left prime, then $\mathcal{B}$ is SDV-representable if and only if $P$ is zero right prime and $\Delta$ has cw-unital determinant.

Proof: Suppose that $R=P \Delta F$, where $P$ is zero right prime, $\Delta$ is cw-unital matrix and $F$ is factor left prime, is a representation of $\mathcal{B}$. Since $P$ is zero right prime there exists $N$ such that $U=\left[\begin{array}{ll}P & N\end{array}\right]$ is unimodular and

$$
R=\left[\begin{array}{ll}
P & N
\end{array}\right]\left[\begin{array}{c}
\Delta F \\
0
\end{array}\right] \text {. }
$$

Considering $U^{-1}=\left[\begin{array}{c}U_{1} \\ U_{2}\end{array}\right]$

$$
U_{1} R=\Delta F \text {. }
$$

So, $\Delta F$ is a representation of $\mathcal{B}$. Therefore $\mathcal{B}$ is SDVrepresentable, by Proposition 4.

Reciprocally, let us suppose that $\mathcal{B}$ is SDV-representable and that $R=\operatorname{ker}(P \Delta F)$, where $P, g \times r$, is factor right prime, $\Delta, r \times r$, is non-singular and $F, r \times q$, is factor left prime is a representation of $\mathcal{B}$. Since $\mathcal{B}$ is SDV-representable there exists a full row rank representation $\tilde{R}$ (with rank $r$ ) such that

$$
\tilde{R}=M R_{c}
$$

where $M$ is a $r \times r$ matrix with cw-unital determinant and $R_{c}$, $r \times q$, factor left prime representation of $\mathcal{B}^{c}$, the controllable part of $\mathcal{B}$. On the other hand, $F$ is also a representation of $\mathcal{B}^{c}$, so there exists $U$, unimodular, such that $F=U R_{c}$. Thus,

$$
R=P \bar{\Delta} R_{c}, \text { where } \bar{\Delta}=U \Delta .
$$

Because $R$ and $\tilde{R}$ are both representations of $\mathcal{B}$, there exist L-polynomial matrices $L$ and $\tilde{L}$ such that

$$
\begin{aligned}
R & =L \tilde{R} \\
\tilde{R} & =\tilde{L} R
\end{aligned}
$$

Thus, from (7), $M R_{c}=\tilde{L} P \bar{\Delta} R_{c}$, that is, (because $R_{c}$ is full row rank),

$$
M=\tilde{L} P \bar{\Delta}
$$

Also, from (6), $P \bar{\Delta} R_{c}=L M R_{c}$ and consequently

$$
P \bar{\Delta}=L M \text {. }
$$

This, together with (8), yields

$$
P \bar{\Delta}=L \tilde{L} P \bar{\Delta} \text {. }
$$


Therefore, $P=L(\tilde{L} P)$. As $P$ is factor right prime and $\tilde{L} P$ is a square factor of $P, \tilde{L} P$ must be unimodular. Hence $P$ is zero right prime. Moreover, recalling that $\operatorname{det} M$ is cw-unital, from $(8), \operatorname{det}(\bar{\Delta})$ is cw-unital. Consequently, also $\operatorname{det}(\Delta)$ is cw-unital.

\section{The REPRESENTATION CONSTRUCTION}

In the previous section, we have shown that a behavior is SDV-representable if and only if any of its representations admits a factorization into a product of a zero right prime matrix by a square matrix with $\mathrm{cw}$-unital determinant by a factor left prime matrix. In this section, we propose a method to construct an SDV-represention for a behavior starting from one of its representations using the referred factorization.

Let $\mathcal{B}=\operatorname{ker} R$ and consider a factorization of $R$ into the following form

$$
R=P \Delta F,
$$

where $P, g \times r$, is factor right prime, $\Delta, r \times r$, is a nonsingular square matrix and $F, r \times q$, is factor left prime. If $P$ is not zero right prime or $\operatorname{det}(\Delta)$ is not cw-unital, then $\mathcal{B}$ is not SDV-representable, else $\mathcal{B}$ is SDV-representable and $\mathcal{B}=\operatorname{ker}(\Delta F)$. In this case, as shown in the proof of Lemma 5 , there exist $F_{1}$ such that

$$
\left[\begin{array}{c}
\Delta F \\
F_{1}
\end{array}\right]
$$

has cw-unital determinant. Moreover,

$$
\left[\begin{array}{c}
\Delta F \\
F_{1}
\end{array}\right] w=\left[\begin{array}{l}
0 \\
I
\end{array}\right] v
$$

is an latent variable representation of $\mathcal{B}$. Since

$$
\operatorname{det}\left(\left[\begin{array}{c}
\Delta F \\
F_{1}
\end{array}\right]\right)
$$

is cw-unital, there exists an unimodular matrix $U$ such that

$$
\bar{R}=U\left[\begin{array}{c}
\Delta F \\
F_{1}
\end{array}\right]
$$

has its support in the third quarter plane and unit independent term. Considering $U=\left[\begin{array}{cc}U_{1} & U_{2}\end{array}\right]$, we have the following latent variable representation of $\mathcal{B}$

$$
\bar{R} w=U_{2} v
$$

Notice that, without loss of generality, we may take $U_{2}$ such that its support also lies in the third quarter plane and zero independent term. In fact, if such does not happen, we multiply $U_{2}$ by $s_{1}^{-p_{1}} s_{2}^{-p_{2}}$, for suitable $p_{1}, p_{2} \in \mathbb{N}$, and take an other driving variable $\bar{v}=\sigma_{1}^{p_{1}} \sigma_{2}^{p_{2}} v$. From (10) it is possible to construct an SDV-representation for $\mathcal{B}$ using some extra auxiliary variables (the states), performing a rather technical, but easy to follow, procedure, that we describe next.
Consider in (10) the L-polynomial matrices written in the following way

$$
\begin{aligned}
\bar{R}= & I+\bar{R}_{10} s_{1}^{-1}+\bar{R}_{01} s_{2}^{-1}+\ldots \\
& +\bar{R}_{k 0} s_{1}^{-k}+\bar{R}_{k 1} s_{1}^{-k} s_{2}^{-1}+\ldots \bar{R}_{0 k} s_{2}^{-k} \\
U_{2}= & \tilde{U}_{10} s_{1}^{-1}+\tilde{U}_{01} s_{2}^{-1}+\ldots \\
& +\tilde{U}_{l 0} s_{1}^{-1}+\tilde{U}_{l 1} s_{1}^{-l} s_{2}^{-1}+\ldots \tilde{U}_{0 l} s_{2}^{-l}
\end{aligned}
$$

where

$$
\begin{aligned}
& k=\max _{(i, j) \in \operatorname{supp}(\bar{R})}\{-i-j\}, \\
& l=\max _{(i, j) \in \operatorname{supp}\left(U_{2}\right)}\{-i-j\}, \\
& \bar{R}_{i j}, \text { for } i, j=1 \ldots, k, \text { are } r \times r \text { real matrices, } \\
& \tilde{U}_{i j}, \text { for } i, j=0 \ldots, l, \text { are } r \times(q-r) \text { real matrices, }
\end{aligned}
$$

and the L-monomials are taken in the degree-lexicographic order.

Take $w$ and define the following $\frac{k(k+1)}{2}$ auxiliary variables:

$$
\begin{aligned}
& \bar{x}_{00}=w \\
& \left.\begin{array}{l}
\bar{x}_{10}=\sigma_{1}^{-1} \bar{x}_{00} \\
\bar{x}_{01}=\sigma_{2}^{-1} \bar{x}_{00}
\end{array}\right\} 2 \\
& \left.\begin{array}{rll}
\bar{x}_{i 0} & = & \sigma_{1}^{-1} \bar{x}_{(i-1) 0} \\
\bar{x}_{(i-1) 1} & = & \sigma_{1}^{-1} \bar{x}_{(i-2) 1} \\
& \vdots & \\
\bar{x}_{1(i-1)} & = & \sigma_{1}^{-1} \bar{x}_{0(i-1)} \\
\bar{x}_{0 i} & = & \sigma_{2}^{-1} \bar{x}_{0(i-1)}
\end{array}\right\} i, \quad \text { for } i=3,4, \ldots, k .
\end{aligned}
$$

Similarly, if $l>1$, take $v$ and define some extra $\frac{(l-1)(l+2)}{2}$ auxiliary variables:

$$
\left.\begin{array}{rl}
\tilde{x}_{10} & =\sigma_{1}^{-1} v \\
\tilde{x}_{01} & =\sigma_{2}^{-1} v
\end{array}\right\} \quad 2
$$


Considering the state variables

$$
x=\left[\begin{array}{c}
\bar{x} \\
\tilde{x}
\end{array}\right],
$$

where

$$
\begin{aligned}
& \bar{x}=\operatorname{col}\left(\bar{x}_{00}, \bar{x}_{10}, \bar{x}_{01}, \ldots, \bar{x}_{(k-1) 0}, \ldots, \bar{x}_{1(k-2)}, \bar{x}_{0(k-1)}\right) \\
& \tilde{x}=\operatorname{col}\left(\tilde{x}_{10}, \tilde{x}_{01}, \ldots, \tilde{x}_{(l-1) 0}, \ldots, \tilde{x}_{1(l-2)}, \tilde{x}_{0(l-1)}\right)
\end{aligned}
$$

the following SDV representation of $\mathcal{B}$ is obtained (from (10), (11) and (12)):

$$
\left\{\begin{aligned}
{\left[\begin{array}{l}
\bar{x} \\
\tilde{x}
\end{array}\right] } & =\left[\begin{array}{cc}
A_{11} & A_{12} \\
0 & A_{22}
\end{array}\right]\left[\begin{array}{l}
\bar{x} \\
\tilde{x}
\end{array}\right]+\left[\begin{array}{l}
B_{1} \\
B_{2}
\end{array}\right] v \\
w & =\left[\begin{array}{ll}
I_{r} 0 & 0
\end{array}\right]\left[\begin{array}{l}
\bar{x} \\
\tilde{x}
\end{array}\right]
\end{aligned}\right.
$$

where

$$
\begin{aligned}
& \text { where } \begin{aligned}
B_{1}= & {\left[\begin{array}{c}
\tilde{U}_{10} s_{1}^{-1}+\tilde{U}_{01} s_{2}^{-1} \\
0 \\
\vdots \\
0
\end{array}\right] } \\
B_{2}= & {\left[\begin{array}{c}
I_{q-r} s_{1}^{-1} \\
I_{q-r} s_{2}^{-1} \\
0 \\
\vdots \\
0
\end{array}\right] }
\end{aligned}
\end{aligned}
$$

and

$$
\begin{aligned}
& A_{11}=\left[\begin{array}{ccccc|c}
\bar{R}_{1} & \cdots & \bar{R}_{i} & \cdots & \bar{R}_{k-1} & \bar{R}_{k} \\
\hline V_{1} & & & & 0 & 0 \\
& \ddots & & & 0 & \vdots \\
0 & & & \ddots & & \\
& & & & V_{k-1} & 0
\end{array}\right] \\
& A_{12}=\left[\begin{array}{ccccc|c}
\tilde{U}_{2} & \cdots & \tilde{U}_{j} & \cdots & \tilde{U}_{l-1} & \tilde{U}_{l} \\
\hline 0 & & & & 0 & 0 \\
& \ddots & & & 0 & \vdots \\
0 & & & \ddots & & \\
& & & & 0 & 0
\end{array}\right] \\
& A_{22}=\left[\begin{array}{ccccc|c}
0 & \cdots & 0 & \cdots & 0 & 0 \\
0 & \cdots & 0 & \cdots & 0 & 0 \\
\hline W_{2} & & & & 0 & 0 \\
& \ddots & & & 0 & \vdots \\
& & W_{j} & & & \\
0 & & & \ddots & & \\
& & & & W_{l-1} & 0
\end{array}\right]
\end{aligned}
$$

with

$$
\begin{array}{r}
\bar{R}_{i}=-\left[\bar{R}_{i 0} s_{1}^{-1}|\cdots| \bar{R}_{2(i-2)} s_{1}^{-1} \mid \bar{R}_{1(i-1)} s_{1}^{-1}+\bar{R}_{0 i} s_{2}^{-1}\right], \\
i=1, \ldots, k
\end{array}
$$

$$
\begin{aligned}
& V_{1}=\left[\begin{array}{c}
s_{1}^{-1} I_{q} \\
s_{2}^{-1} I_{q}
\end{array}\right] \\
& V_{i}=\left[\begin{array}{c|c}
s_{1}^{-1} I_{i q} & 0 \\
\hline 0 & V_{1}
\end{array}\right], i=2, \ldots, k \\
& \tilde{U}_{j}=\left[\tilde{U}_{j 0} s_{1}^{-1}|\ldots| \tilde{U}_{2(j-2)} s_{1}^{-1} \mid \tilde{U}_{1(j-1)} s_{1}^{-1}+\tilde{U}_{0 j} s_{2}^{-1}\right], \\
& j=2, \ldots, l
\end{aligned}
$$

$W_{j}=\left[\begin{array}{c|c}s_{1}^{-1} I_{j(q-r)} & 0 \\ \hline 0 & W_{1}\end{array}\right], j=2, \ldots, l$.

Note that, if $l=0,1$ the variables $\tilde{x}$ are not considered and consequently the blocks $A_{22}$ and $B_{2}$ do not appear.

\section{FINAL REMARKS}

In this paper we have proposed a strategy for testing if a 2D kernel behavior is SDV-representable. More concretely, we have shown that if a kernel representation of a behavior is decomposed as a product of a factor right prime matrix by a non-singular square matrix by a factor left prime matrix, then the behavior is SDV-representable if and only if the factor right prime matrix is indeed zero right prime and the square matrix has cw-unital determinant. This representability test improves a previous result obtained in [2], as it is independent from the chosen behavior representation. The obtained decomposition allows to set up an algorithm for the construction of 2D SVD representations.

\section{REFERENCES}

[1] I. Brás and P. Rocha, "State/driving-variable representation of 2D systems," Multidimensional Systems and Signal Processing, vol. 13, pp. 129-156, 2002.

[2] _ "A test for state/driving-variable representability of 2D behaviors," in Constructive Algebra and Systems Theory, B. Hanzon and M. Hazewinkel, Eds. Royal Netherlands Academy of Arts and Sciences, 2006, pp. 185-192.

[3] E. Fornasini and G. Marchesini, "Doubly-indexed dynamical systems: State-space models and structural properties," Mathematical Systems Theory, vol. 12, pp. 59-72, 1978.

[4] P. Rocha, "Structure and representation 2-D systems," Ph.D. dissertation, Rijkuniversiteit Groningen, 1990.

[5] E. Fornasini, P. Rocha, and S. Zampieri, "State space realization of 2D finite-dimensional behaviours," SIAM J. Control and Optimization, vol. 31 , no. 6, pp. 1502-1517, 1993.

[6] U. Oberst, "Multidimensional constant linear systems," Acta Applicandae Mathematicae, vol. 20, pp. 1-175, 1990.

[7] M. E. Valcher, "On the decomposition of two-dimensional behaviors," Multidimensional Syst. Signal Process, vol. 11, no. 1-2, pp. 49-65, 2000.

[8] E. Zerz and V. Lomadze, "A constructive solution to interconnection and decomposition problems with multidimensional behaviors," SIAM J. Control and Optimization, vol. 40, no. 4, pp. 1072-1086, 2001.

[9] D. C. Youla and G. Gnavi, "Notes on n-dimensional system theory," IEEE Trans. on Circuits and Systems, no. 2, pp. 105-111, Feb. 1979.

[10] J. C. Willems, "Models for dynamics," Dynamics Reported, vol. 2, pp. 171-269, 1989. 